\title{
A Randomized Phase 1 Dose Escalation Study to Evaluate Safety, Tolerability, and Pharmacokinetics of Trabodenoson in Healthy Adult Volunteers
}

\author{
Alan Laties, Cadmus C. Rich, Randall Stoltz, Vernon Humbert, \\ Chaim Brickman, William McVicar, and Rudolf A. Baumgartner ${ }^{2}$
}

\begin{abstract}
Purpose: To investigate the safety, tolerability, and pharmacokinetics of trabodenoson, a highly selective adenosine mimetic targeting the adenosine $\mathrm{A}_{1}$ receptor.

Methods: In Part 1, 60 healthy adult volunteers were randomized to 14 days of twice-daily topical monocular application of placebo or trabodenoson $(200,400,800,1,600,2,400$, or 3,200 $\mu \mathrm{g})$. In Part 2, 10 subjects were randomized to placebo or 8 escalating doses of bilateral trabodenoson (total daily doses: 1,800-6,400 $\mu \mathrm{g}$ ).

Results: The incidence of treatment-related adverse events in Part 1 was similar in the trabodenoson (27.8\%) and placebo (25.0\%) groups. Most were mild in intensity. The most common adverse events (AEs) for trabodenoson and placebo were headache $(25.0 \%$ vs. $33 \%$, respectively) and eye pain $(11.1 \%$ vs. $4.2 \%$, respectively). Ocular AEs were infrequent (16.7\% and $17.9 \%$, respectively), were self-limited, lasted $<24 \mathrm{~h}$, and were typically mild in intensity. The most common ocular AE was eye pain (9.5\% and 3.6\%, respectively), with a single observation of ocular hyperemia ( $200 \mu \mathrm{g}$ trabodenoson). Trabodenoson was rapidly absorbed [median time to maximum concentration $\left(\mathrm{t}_{\max }\right): \sim 0.08$ to $\left.0.27 \mathrm{~h}\right]$ and eliminated $\left(\mathrm{t}_{1 / 2}: 0.48-2.0 \mathrm{~h}\right)$, with no evidence of drug accumulation. Systemic exposure to topical trabodenoson was dose related but not dose proportional, with a plateau effect at doses $\geq 2,400 \mathrm{mg}$ per eye. No clinically significant treatment-related systemic AEs were observed, and increasing systemic exposure had no effect on heart rate or blood pressure.

Conclusions: Ocular doses of trabodenoson up to 3,200 $\mu \mathrm{g}$ per eye were safe and well tolerated in the eye and resulted in no detectable systemic effects in healthy adult volunteers.
\end{abstract}

Keywords: primary open angle glaucoma, ocular hypertension, intraocular pressure, Trabodenoson, adenosine, trabecular meshwork

\section{Introduction}

$\mathbf{E}$ LEVATED INTRAOCULAR PRESSURE (IOP) is an established risk factor for the development and progression of primary open-angle glaucoma, and reduction of IOP is the only therapeutic intervention known to lower the risk of developing glaucoma ${ }^{1,2}$ and slow its progression. ${ }^{3,4}$ Reduction of IOP can be achieved with medication, laser therapy, or surgical intervention. Medications delivered as topical eye drops are the preferred first-line therapy for the management of ocular hypertension and primary open-angle glaucoma. Approved medications lower IOP by reducing the rate of aqueous humor production within the eye or increasing the rate of aqueous humor egress from the eye.

Many patients with ocular hypertension or glaucoma require more than 1 medication in an effort to achieve a target IOP reduction. ${ }^{5}$ Following a period of innovation in the 1990 s with the introduction of topical carbonic anhydrase inhibitors, alpha-adrenergic agonists, and prostaglandin analogs into the US marketplace, no novel IOP-lowering drug

\footnotetext{
${ }^{1}$ Scheie Eye Institute, Philadelphia, Pennsylvania.

${ }^{2}$ Inotek Pharmaceuticals Corporation, Lexington, Massachusetts.

${ }^{3}$ Covance Global Clinical Pharmacology, Evansville, Indiana.

${ }^{4}$ Evansville Heart Center, Evansville, Indiana.
}

(C)Alan Laties, et al., 2016; Published by Mary Ann Liebert, Inc. This Open Access article is distributed under the terms of the Creative Commons Attribution Noncommercial License (http://creativecommons.org/licenses/by-nc/4.0/) which permits any noncommercial use, distribution, and reproduction in any medium, provided the original author(s) and the source are credited. 
class has obtained regulatory approval in the United States. An unmet need remains for a medication with a novel mechanism of action that complements the actions of available therapies. As existing therapies are not devoid of side effects, an ideal drug candidate would have an excellent safety profile (ocular and systemic) 1) to facilitate its use as a first-line monotherapy, 2) to be safely added to existing medical therapies to further reduce IOP, and/or 3) to be combined with another medical therapy in a fixed dose combination without significant added side effects.

Adenosine is a naturally occurring purine nucleoside that modulates many physiologic processes. Cellular signaling by adenosine occurs through 4 known adenosine receptor subtypes $\left(A_{1}, A_{2 A}, A_{2 B}\right.$, and $\left.A_{3}\right)$. Accumulating evidence has begun to clarify the role of adenosine and its receptors in modulating IOP. ${ }^{6,7}$ Selective $\mathrm{A}_{1}$ receptor agonism has been shown to lower IOP in mouse, rabbit, monkey, and cat animal models, ${ }^{7-12}$ while selective agonism of $\mathrm{A}_{2 \mathrm{~A}}$ and $\mathrm{A}_{3}$ receptors and nonselective adenosine mimetics can elevate IOP. ${ }^{7}$ Elevated endogenous adenosine concentrations in the aqueous humor of ocular hypertensive (but not normotensive) patients suggest a local role for adenosine in IOP homeostasis, ${ }^{13}$ and the adenosine $A_{1}$ receptor may be a specific target for IOP modulation in ocular hypertension and glaucoma patients.

Trabodenoson is an adenosine mimetic that has a high affinity $(\mathrm{Ki}=\sim 1 \mathrm{nM})$ and specificity for the adenosine $\mathrm{A}_{1}$ receptor. A topical ophthalmic formulation of trabodenoson is in clinical development for the reduction of elevated IOP in patients with ocular hypertension or primary open-angle glaucoma. In this article, we present the results of a Phase 1 clinical trial to assess the tolerability and safety of trabodenoson in healthy adult subjects.

\section{Methods}

This was a randomized, double-masked, placebo-controlled dose escalation study designed to characterize the systemic and local ocular safety and tolerability of trabodenoson delivered via topical ophthalmic dosing. The study was registered (ClinicalTrials.gov identifier: NCT01123772) and was conducted in accordance with the Good Clinical Practices and the Declaration of Helsinki. The study was conducted at a single site (Covance Clinical Research Unit, Evansville, IN). The protocol was approved by the site's Institutional Review Board, and all subjects provided written informed consent.

\section{Subjects}

The study was conducted in 2 parts. In Part 1, 60 subjects were sequentially enrolled into 6 cohorts of 10 subjects each (Cohorts 1-6), and in Part 2, 10 subjects were enrolled in a single cohort (Cohort 7). Subjects were healthy volunteers aged 35-65 years, with a body weight range of $50-115 \mathrm{~kg}$. Key exclusion criteria include best-corrected visual acuity (BCVA) below 20/60 in either eye, any active eye disease, narrow or closed anterior chamber angles, history of central retinal vein occlusion, visual field loss, or ocular surgery or trauma, pregnancy, significant systemic disease (including renal disease, cardiac disease excluding controlled systemic hypertension, asthma, or chronic obstructive pulmonary disease), and current or recent use of certain medications (calcium channel blockers, beta-blockers, sympathomimetics, oral carbonic anhydrase inhibitors, dipyridamole or other adenosine antagonists, or any experimental agents).

\section{Enrollment and randomization}

All subjects attended a screening visit during which eligibility was evaluated via a comprehensive review of medical history and an ophthalmological examination. Subjects were enrolled into the 7 cohorts of 10 subjects each and were randomized in a 3:2 ratio of trabodenoson:placebo using a permuted block strategy to ensure that each of the cohorts included 6 subjects receiving active drug and 4 receiving placebo. This randomization scheme was chosen to increase the number of actively treated subjects while still maintaining an appropriate control number to clearly understand the safety profile of trabodenoson.

\section{Treatments}

Subjects randomized to active treatment received topical ophthalmic trabodenoson in doses varying from 200 to $3,200 \mu \mathrm{g}$ per dose. Subjects randomized to placebo received the trabodenoson vehicle. Subjects in Cohorts 1-6 (Part 1) received unilateral dosing in the study eye twice daily for 14 days, with doses beginning at $200 \mu \mathrm{g}$ for Cohort 1 and $400,800,1,600,2,400$, and $3,200 \mu \mathrm{g}$, respectively, for Cohorts 2-6. Subjects assigned to Cohort 7 (Part 2) received bilateral topical trabodenoson once daily beginning at $200 \mu \mathrm{g}$ in 1 eye and $1,600 \mu \mathrm{g}$ in the fellow eye $(200 / 1,600)$ on Day 1 and every other day thereafter with dose escalation, if tolerated, every other day as follows: 400/1,600 on Day 3, $800 / 1,600$ on Day 5, 1,600/1,600 on Day 7, 2,400/1,600 on Day $9,2,400 / 2,400$ on Day $11,3,200 / 2,400$ on Day 13 , and 3,200/3,200 on Day 15, the highest single-eye dose from Part 1. On days when the doses were unequal, the right eye received the higher dose. Prespecified stopping rulesincorporating clinical, laboratory, and cardiac data-governed the continuation of the study from one dose to the next in both Parts 1 and 2 .

\section{Schedule of visits}

The study schedule included a screening visit, a 2-day inpatient pretreatment baseline assessment period (during which a placebo run-in was initiated), a 14-day (Cohorts 16) or 15-day (Cohort 7) inpatient treatment period, and a 6day (Cohorts 1-6) or 5-day (Cohort 7) postdosing outpatient observation period.

\section{Assessments}

The safety and tolerability of trabodenoson were assessed on the basis of both clinical findings and adverse event (AE) monitoring conducted during the pretreatment baseline period and at selected time points during the treatment period. AEs were categorized by System Organ Class (SOC) and Preferred Terms using the Medical Dictionary for Regulatory Activities (MedDRA, version 13). All AEs were graded by severity (mild, moderate, and severe) and likely causal relationship to the study drug (possibly, probably, and definitely).

Systemic safety assessments included physical examinations, vital signs, and orthostatic blood pressure, and clinical laboratory tests included complete blood counts and blood chemistry assessments as well as renal, hepatic, and cardiac biomarkers and urinalysis. Specific cardiovascular safety assessments included 12-lead electrocardiograms (ECGs), 
48-h cardiac Holter monitoring, cardiac rhythm strips, continuous multilead cardiac telemetry monitoring, and troponin levels. The ECG and Holter monitoring data were centrally read in a masked manner (ERT, Philadelphia, PA). The effect of trabodenoson on bronchoconstriction was evaluated using expiratory flow rates for the forced expiratory volume in the first second $\left(\mathrm{FEV}_{1}\right)$, and the effect of trabodenoson on sleepiness, lethargy, or tiredness was assessed using the Karolinska Sleepiness Scale.

Ocular safety assessments included BCVA, IOP, examination of the external eye (periorbital area, eyelids, eyelashes, conjunctiva, sclera, and pupils), slit-lamp examination (eyelids, eyelashes, conjunctiva, cornea, sclera, pupils, iris, and lens), and dilated (and undilated at some time points) fundoscopic examination. BCVA was assessed using the Early Treatment Diabetic Retinopathy Study (ETDRS) protocol. IOP was measured using Goldmann applanation tonometry in a masked manner.

Pharmacokinetic (PK) parameters calculated included area under the concentration-time curve (AUC), maximum concentration $\left(\mathrm{C}_{\max }\right)$, time to $\mathrm{C}_{\max }\left(\mathrm{t}_{\max }\right)$, and plasma halflife $\left(t_{1 / 2}\right)$. Blood samples were collected at multiple time points for these evaluations.

\section{Statistical analysis}

The primary objective of Part 1 of this study was to evaluate the tolerability and safety of a 14-day topical monocular application of twice-daily trabodenoson 200$3,200 \mu \mathrm{g}$ in healthy adult volunteers. Likewise, the primary objective of Part 2 was to evaluate the medication's tolerability and safety following bilateral application of escalating single doses. The secondary objective included PK analysis of both trabodenoson and its primary metabolite (INO-2446), and the exploratory objective was to evaluate the pharmacodynamic effects of trabodenoson on IOP in this healthy ocular normotensive population. Statistical analysis included descriptive statistics of subject demographics and outcomes based on the intent-to-treat population (all subjects receiving study medication). No inferential statistics were conducted, and therefore, no formal power/sample size calculations were performed.

\section{Results}

\section{Demographics and disposition}

Overall, 70 subjects were enrolled in this study, with 10 in each of the 7 cohorts. Demographic and baseline characteristics were generally similar across the different treatment groups in both Part 1 and Part 2 of the study (Table 1). The mean age across cohorts and treatment groups ranged from 45 to 52 years; $50 \%-83 \%$ of subjects were male, and $33 \%-$ $88 \%$ were white. Observed differences were not considered to be clinically relevant.

In Part 1, 56 of 60 subjects completed the study. One subject assigned to trabodenoson treatment discontinued therapy due to increases in the liver enzymes aspartate aminotransferase, alanine transaminase, and alkaline phosphatase and was subsequently diagnosed with chronic cholecystitis due to cholelithiasis. Because symptoms of recurrent right upper quadrant and right flank pain predated study enrollment, this finding was deemed unrelated to study drug. Three subjects assigned to placebo discontinued

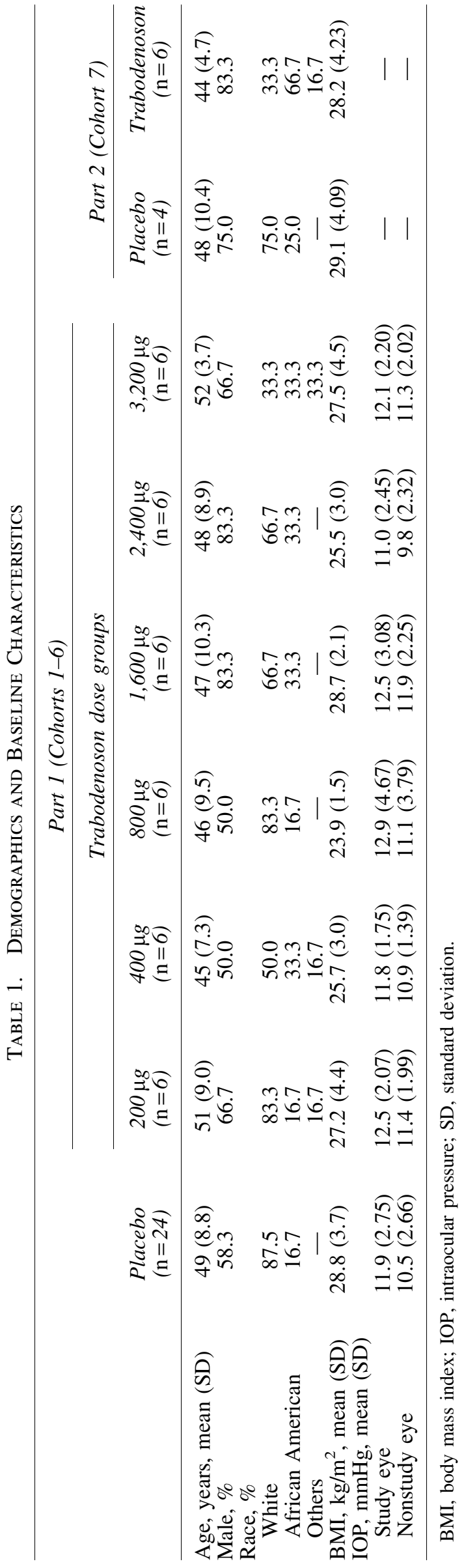


Table 2. Overall Summary of AEs, $N(\%)$

\begin{tabular}{|c|c|c|c|c|}
\hline & \multicolumn{2}{|c|}{ Part 1 (Cohorts 1-6) } & \multicolumn{2}{|c|}{ Part 2 (Cohort 7) } \\
\hline & Placebo $(\mathrm{n}=24)$ & Trabodenoson $(\mathrm{n}=36)$ & Placebo $(\mathrm{n}=4)$ & Trabodenoson $(\mathrm{n}=6)$ \\
\hline Any AE & $21(87.5)$ & $18(50.0)$ & $1(25.0)$ & $3(50.0)$ \\
\hline Related AE & $6(25.0)$ & $10(27.8)$ & $1(25.0)$ & $1(16.7)$ \\
\hline Serious $\mathrm{AE}^{\mathrm{a}}$ & $1(4.2)$ & - & - & - \\
\hline $\mathrm{AE}$ resulting in discontinuation & $2(8.3)$ & $1(2.8)^{\mathrm{b}}$ & - & - \\
\hline
\end{tabular}

${ }^{a}$ One serious $\mathrm{AE}$ of mild supraventricular tachycardia occurred in a subject assigned to placebo in Cohorts 1-6 and led to study discontinuation.

${ }^{\mathrm{b}}$ One subject assigned to trabodenoson in cohorts 1-6 was discontinued due to active chronic cholecystitis.

$\mathrm{AE}$, adverse event.

therapy. One developed a panic attack and supraventricular tachycardia, another developed a toothache requiring discharge from the research unit to seek care and one for more extensive follow-up of abnormal ECG and cardiac telemetry data identified during the baseline period. In Part 2, all 10 enrolled subjects completed the study.

\section{Extent of exposure to trabodenoson}

All subjects randomized to trabodenoson in Cohorts 1-6 received all scheduled administrations of study drug, with the exception of 1 subject randomized to $2,400 \mu \mathrm{g}$ trabodenoson, who was withdrawn from the study due to unrelated cholecystitis. Therefore, this cohort of subjects received 93.2\% (151/162) of scheduled administrations. Cumulative drug exposure in Cohorts 1-6 ranged from $32,400 \mu \mathrm{g}$ in the $200 \mu \mathrm{g}$ Cohort 1 to $518,400 \mu \mathrm{g}$ in the $3,200 \mu \mathrm{g}$ cohort. Subjects in Cohort 7 received all scheduled trabodenoson administrations for a total of $181,200 \mu \mathrm{g}$ in the solitary $6,400 \mu \mathrm{g}$ cohort.

\section{Safety results}

All AEs. The overall frequency of AEs is given in Table 2. In Cohorts 1-6, the proportion of subjects with AEs was higher in the placebo group $(87.5 \%, 21 / 24$ subjects) than in the trabodenoson group (50.0\%, 18/36 subjects). Common AEs occurring at a rate $\geq 5 \%$ in Cohorts $1-6$ are presented in Table 3 and included headache, eye pain, back pain, dermatitis, and excoriation, all of which were more frequent in the placebo group than in the combined trabodenoson groups, except for eye pain $(4.2 \%$ vs. $11 \%)$ and dermatitis (0\% vs. $5.6 \%)$.

The proportion of subjects with AEs in Cohort 7 (Table 3) was lower in the placebo group $(25.0 \%, 1 / 4$ subjects) than in the trabodenoson group $(50.0 \%, 3 / 6$ subjects). A single subject in the placebo group experienced cheilitis (inflammation of the lips), photophobia, excoriation, and pruritus. In the trabodenoson group, one subject experienced constipation at the 1,600/800 dose level; another experienced headache as well as a hematoma and pain at the site of a PK blood draw at the 1,600/200 dose level, and a third subject experienced headache at the 3,200/2,400 dose level.

Treatment-related AEs. The proportion of subjects experiencing treatment-related AEs was similar between the placebo and trabodenoson groups $(25.0 \%$ vs. $27.8 \%$, respectively). In the placebo group, 6 subjects experienced 6 AEs judged by the investigator to be treatment related, including 1 case each of eye pain, eye pruritus, visual

Table 3. Common AEs (Overall Preferred Term $\geq 5 \%$ ), N (\%)

\begin{tabular}{|c|c|c|c|c|c|c|c|c|}
\hline \multirow[b]{2}{*}{$\begin{array}{l}\text { System Organ Class } \\
\text { Preferred Term }\end{array}$} & \multirow[b]{2}{*}{$\begin{array}{l}\text { Placebo } \\
(\mathrm{n}=24)\end{array}$} & \multicolumn{6}{|c|}{ Part 1 (Cohorts 1-6) trabodenoson dose groups } & \multirow[b]{2}{*}{$\begin{array}{l}\text { Overall } \\
\mathrm{N}=36\end{array}$} \\
\hline & & $\begin{array}{l}200 \mu g \\
(\mathrm{n}=6)\end{array}$ & $\begin{array}{c}400 \mu g \\
(\mathrm{n}=6)\end{array}$ & $\begin{array}{l}800 \mu g \\
(\mathrm{n}=6)\end{array}$ & $\begin{array}{c}1,600 \mu g \\
(\mathrm{n}=6)\end{array}$ & $\begin{array}{c}2,400 \mu g \\
(\mathrm{n}=6)\end{array}$ & $\begin{array}{c}3,200 \mu g \\
(\mathrm{n}=6)\end{array}$ & \\
\hline \multicolumn{9}{|l|}{ Nervous system disorders } \\
\hline $\begin{array}{c}\text { Headache } \\
\text { Eye disorders }\end{array}$ & $8(33.3)$ & $4(66.7)$ & $1(16.7)$ & $1(16.7)$ & $1(16.7)$ & $1(16.7)$ & $1(16.7)$ & $9(25.0)$ \\
\hline Eye pain & $1(4.2)$ & $2(33.3)$ & $1(16.7)$ & - & - & - & $1(16.7)$ & $4(11.1)$ \\
\hline Photophobia & - & - & - & - & - & - & - & - \\
\hline Pruritus & - & - & - & - & - & - & - & - \\
\hline \multicolumn{9}{|c|}{ Musculoskeletal and connective tissue disorders } \\
\hline Back pain & $4(16.7)$ & - & - & $1(16.7)$ & - & $1(16.7)$ & - & $2(5.6)$ \\
\hline \multicolumn{9}{|c|}{ Skin and subcutaneous tissue disorders } \\
\hline Cheilitis & - & - & - & - & - & - & - & - \\
\hline Dermatitis & - & - & - & $1(16.7)$ & - & - & $1(16.7)$ & $2(5.6)$ \\
\hline \multicolumn{9}{|l|}{ Gastrointestinal disorders } \\
\hline Constipation & - & - & - & - & - & - & - & - \\
\hline \multicolumn{9}{|c|}{ Injury, poisoning, and procedural complications } \\
\hline Excoriation & $4(16.7)$ & $1(16.7)$ & - & $1(16.7)$ & - & - & $1(16.7)$ & $3(8.3)$ \\
\hline Vessel puncture site hematoma & - & - & - & - & - & - & - & - \\
\hline Vessel puncture site pain & - & - & - & - & - & - & - & - \\
\hline
\end{tabular}


Table 4. Ocular AEs: Cohorts $1-7, N(\%)$

\begin{tabular}{|c|c|c|c|c|c|c|c|c|c|c|}
\hline \multirow[b]{2}{*}{$\begin{array}{l}\text { System Organ Class } \\
\text { Preferred Term }\end{array}$} & \multirow[b]{2}{*}{$\begin{array}{l}\text { Placebo } \\
(\mathrm{n}=24)\end{array}$} & \multicolumn{7}{|c|}{ Part 1 (Cohorts 1-6) } & \multicolumn{2}{|c|}{ Part 2 (Cohort 7) } \\
\hline & & $\begin{array}{l}200 \mu g \\
(\mathrm{n}=6)\end{array}$ & $\begin{array}{l}400 \mu g \\
(\mathrm{n}=6)\end{array}$ & $\begin{array}{l}800 \mu g \\
(\mathrm{n}=6)\end{array}$ & $\begin{array}{c}1,600 \mu g \\
(\mathrm{n}=6)\end{array}$ & $\begin{array}{c}2,400 \mu g \\
(\mathrm{n}=6)\end{array}$ & $\begin{array}{c}3,200 \mu g \\
(\mathrm{n}=6)\end{array}$ & $\begin{array}{l}\text { Overall } \\
(\mathrm{n}=36)\end{array}$ & $\begin{array}{c}\text { Placebo } \\
(\mathrm{n}=4)\end{array}$ & $\begin{array}{l}\text { Overal } \\
(\mathrm{n}=6)\end{array}$ \\
\hline Eye pain & $1(4.2)$ & $2(33.3)$ & $1(16.7)$ & - & - & - & $1(16.7)$ & $4(11.1)$ & - & - \\
\hline Eye pruritus & $1(4.2)$ & - & - & - & - & - & $1(16.7)$ & - & - & - \\
\hline Hordeolum & - & - & - & $1(16.7)$ & - & - & - & - & - & - \\
\hline Photophobia & - & - & - & - & - & - & - & - & $1(25)$ & - \\
\hline $\begin{array}{l}\text { Papular rash (lower } \\
\text { right eyelid) }\end{array}$ & $1(4.2)$ & - & - & - & - & - & - & - & - & - \\
\hline $\begin{array}{l}\text { Conjunctival } \\
\text { hyperemia }\end{array}$ & - & $1(16.7)$ & - & - & - & - & - & - & - & - \\
\hline Visual impairment & $1(4.2)$ & - & - & - & - & - & - & - & - & - \\
\hline
\end{tabular}

impairment, fatigue, acne, and papular rash. In the combined trabodenoson groups, 10 subjects experienced 15 treatmentrelated AEs, including 7 cases of headache, 4 cases of eye pain, and 1 case each of eye pruritus, conjunctival hyperemia, fatigue, and rhinorrhea. All but 2 of these were graded as mild in severity: 1 case each of headache and conjunctival hyperemia, both in subjects assigned to the $200 \mu \mathrm{g}$ group, were graded as moderate.

In Cohort 7, only 1 subject each in the placebo and trabodenoson groups $(25.0 \%$ and $16.7 \%$, respectively) experienced treatment-related AEs: 1 case of headache in a subject in the $200 \mu \mathrm{g}$ trabodenoson group graded as mild and 1 case of photophobia in the placebo group graded as mild. Both resolved spontaneously without discontinuation of study medication.

Serious AEs. The only serious adverse event in the study (supraventricular tachycardia) was experienced by a subject in the placebo group who discontinued treatment.

Ocular AEs. Ocular AEs were uncommon, lasted less than $24 \mathrm{~h}$, were self-limited, and were usually mild in intensity. No ocular AE in any cohort was deemed serious by the investigator. In Cohorts 1-6, 4 subjects (16.7\%) experienced ocular AEs (1 case each of pain, pruritus, papular rash of the eyelid, photophobia, and visual impairment) compared to 7 subjects $(16.7 \%)$ in the combined trabodenoson groups (4 cases of eye pain and 1 case each of pruritus, hordeolum, and conjunctival hyperemia). In Cohort 7 , 1 subject in the placebo group experienced photophobia, and no subjects in the trabodenoson group experienced an ocular AE. No anterior chamber inflammation was noted in any subject. No clinically significant changes in visual acuity were noted in any subjects. These results are summarized in Table 4.

Other safety evaluations. No clinically significant treatment-related abnormalities were observed in clinical laboratory studies, ocular or physical examinations, pulmonary or cardiac function tests, or sleepiness assessments in any cohort.

\section{PK results}

The PK data demonstrated that the exposure to trabodenoson and its main metabolite INO-2446 increased dose- proportionally between the 200 and $800 \mu \mathrm{g}$ dose groups, while at higher doses, drug exposure generally increased in a dose-dependent but not dose-proportional manner. Specifically, at the highest 3 doses administered in Cohort 7, there was no apparent increase in systemic exposure with increasing topical ocular dose (Fig. 1). This plateau effect suggests that little additional drug is absorbed into the systemic circulation following doses above $4,800 \mu \mathrm{g}(2,400 \mu \mathrm{g}$ per eye). The median $t_{\max }$ for trabodenoson occurred at $\sim 0.08$ to $0.27 \mathrm{~h}$ across all treatment cohorts, indicating rapid absorption following ocular administration. The mean $t_{1 / 2}$ of trabodenoson ranged from 0.48 to $2.0 \mathrm{~h}$ across all treatment cohorts except for the $3,200 \mu \mathrm{g}$ cohort and demonstrated the rapid elimination from plasma following ocular administration. Two subjects in the $3,200 \mu \mathrm{g}$ cohort had a longer than expected half-life of $\sim 17 \mathrm{~h}$. The reason for this finding is unknown, although a corresponding increase in the half-life of primary metabolite INO-2446 was not observed in these 2 subjects, suggesting that these data may be aberrant. A comparison of the concentrations and exposures for trabodenoson on Day 1 and Day 14 demonstrated a lack of accumulation of drug in plasma following repeated ocular administration at any dose tested. Exposure to the primary metabolite INO-2446, as measured by $\mathrm{C}_{\max }$ and AUC, ranged from $\sim 13 \%$ to $37 \%$ of the corresponding exposures

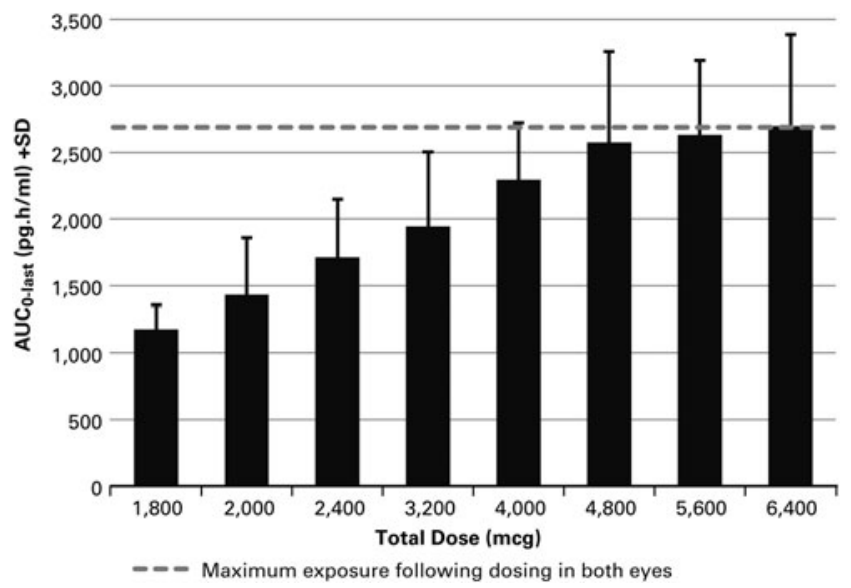

FIG. 1. The relationship between the $\mathrm{AUC}_{\text {last }}$ and trabodenoson dose in Cohort 7 demonstrates a plateau above $2,400 \mu \mathrm{g}$ per eye dosing (total dose $4,800 \mu \mathrm{g}$ ). AUC, area under the concentration-time curve. 
to trabodenoson. Variability of $\mathrm{C}_{\max }$ and $\mathrm{AUC}$ values for INO-2446 were high but similar to those for trabodenoson. The mean $\mathrm{t}_{1 / 2}$ for INO-2446 was $\sim 0.68$ to $5.0 \mathrm{~h}$ and was similar across all dose groups. Increasing systemic exposure had no effect on heart rate or blood pressure.

\section{Discussion}

This study evaluated the local and systemic safety and tolerability of topical ophthalmic administration of trabodenoson in healthy adult volunteers. Escalating ocular doses of trabodenoson far in excess of doses associated with IOP reduction in subjects with primary open-angle glaucoma or ocular hypertension $(500 \mu \mathrm{g})$ were safe and well tolerated, with no dose-limiting toxicities or treatment-related ocular AEs, such as conjunctival hyperemia or anterior chamber inflammation, observed. The incidence of overall AEs was lower in the trabodenoson group $(50 \%)$ than in the placebo group $(87.5 \%)$ for Cohorts $1-6$ in Part 1 of the study. The most commonly reported AEs $(\geq 5 \%)$ in Part 1 were headache, eye pain, back pain, dermatitis, and excoriation. In Part 2 of the study, trabodenoson was associated with a greater incidence of AEs than placebo (50\% vs. $25 \%$, respectively). There was no increase in the frequency of overall AEs with increasing dose of trabodenoson, and the incidence of treatment-related AEs was similar between the trabodenoson and placebo groups in both parts of the study. Most treatment-related AEs were mild in intensity. The most common treatment-related AEs were headache and eye pain. AEs potentially associated with systemic adenosine, such as chest discomfort, flushing, dyspnea, and hypotension, were not observed in this study. Overall, trabodenoson administered via topical ophthalmic application demonstrated favorable systemic safety at doses up to $6,400 \mu \mathrm{g}$ $(3,200 \mu \mathrm{g}$ per eye) per day, the highest practically achievable ocular dose that required 4 consecutive drops of the formulation in each eye, $5 \mathrm{~min}$ apart, at the highest drug concentration available.

The incidence of ocular AEs was similar between trabodenoson and placebo treatment groups, and these AEs were infrequent and self-limited, and most were mild in intensity. Only 1 trabodenoson-related AE of conjunctival hyperemia was reported in the lowest trabodenoson treatment group $(200 \mathrm{mg}$ ); it resolved within $1 \mathrm{~h}$ and did not recur with subsequent dosing. There were no serious ocular AEs. Overall, trabodenoson demonstrated favorable ocular safety and tolerability.

PK analysis demonstrated a dose-proportional increase in plasma exposure up to a dose of $800 \mu \mathrm{g}$ and dose-dependent but not dose-proportional increases in exposure at higher doses, with the highest systemic exposure at the $6,400 \mu \mathrm{g}$ per day dose level in Part 2. The plasma half-life of trabodenoson was generally under $2 \mathrm{~h}$ at all doses, and under $5 \mathrm{~h}$ for its primary metabolite, demonstrating rapid plasma clearance and no drug accumulation with repeated dosing.

This study was designed to thoroughly assess the safety, tolerability, and pharmacokinetics of trabodenoson. However, these rigorous assessments required isolation in a phase 1 unit, necessitating a smaller sample size. Thus, the generalizability of these results to actual patients with glaucoma or ocular hypertension may have limitations. A second study evaluating similar parameters in the target population of adults with ocular hypertension or primary open angle glaucoma can be found in this same journal issue (see pages $\mathrm{XX}-\mathrm{XX}$ ).

In summary, trabodenoson delivered via topical ophthalmic application at doses up to $6,400 \mu \mathrm{g}$ per day demonstrated favorable ocular and systemic safety and tolerability. PK analysis indicated no accumulation with repeated dosing. Additionally, a dose-related increase in plasma drug exposure - that was less than dose proportional and plateaued at doses above $2,400 \mu \mathrm{g}$ per eye-was observed. The profile of this drug candidate may fulfill an unmet need in glaucoma management by providing clinicians and patients with a novel mechanism of action for lowering IOP that is both safe and well tolerated. These results support continued clinical development of trabodenoson to confirm and characterize its safety and efficacy profile in the broader patient population, including patients with primary open-angle glaucoma and ocular hypertension.

\section{Author Disclosure Statement}

A.L. is a consultant and member of the Inotek Scientific Advisory Board. C.C.R., C.B., W.M., and R.A.B. are or were employees of Inotek Pharmaceuticals during the conduct of the work reported herein and own stock and stock options. R.S. and V.H have no competing financial interests.

\section{References}

1. Gordon, M.O., Beiser, J.A., Brandt, J.D., et al. The Ocular Hypertension Treatment Study: baseline factors that predict the onset of primary open-angle glaucoma. Arch. Ophthalmol. 120:714-720; discussion 829-830, 2002.

2. Kass, M.A., Heuer, D.K., Higginbotham, E.J., et al. The Ocular Hypertension Treatment Study: a randomized trial determines that topical ocular hypotensive medication delays or prevents the onset of primary open-angle glaucoma. Arch. Ophthalmol. 120:701-713, 2002.

3. Leske, M.C., Heijl, A., Hussein, M., et al. Factors for glaucoma progression and the effect of treatment: the early manifest glaucoma trial. Arch. Ophthalmol. 121:48-56, 2003.

4. Heijl, A., Leske, M.C., Bengtsson, B., Hyman, L., Bengtsson, B., and Hussein, M. Reduction of intraocular pressure and glaucoma progression: results from the Early Manifest Glaucoma Trial. Arch. Ophthalmol. 120:1268-1279, 2002.

5. Kass, M.A., Gordon, M.O., Gao, F., et al. Delaying treatment of ocular hypertension: the ocular hypertension treatment study. Arch. Ophthalmol. 128:276-287, 2010.

6. Sanderson, J., Dartt, D.A., Trinkaus-Randall, V., et al. Purines in the eye: recent evidence for the physiological and pathological role of purines in the RPE, retinal neurons, astrocytes, Muller cells, lens, trabecular meshwork, cornea and lacrimal gland. Exp. Eye. Res. 127:270-279, 2014.

7. Avila, M.Y., Stone, R.A., and Civan, M.M. A(1)-, A(2A)- and A(3)-subtype adenosine receptors modulate intraocular pressure in the mouse. Br. J. Pharmacol. 134:241-245, 2001.

8. Tian, B., Gabelt, B.T., Crosson, C.E., and Kaufman, P.L. Effects of adenosine agonists on intraocular pressure and aqueous humor dynamics in cynomolgus monkeys. Exp. Eye. Res. 64:979-989, 1997.

9. Crosson, C.E., and Gray, T. Characterization of ocular hypertension induced by adenosine agonists. Invest. Ophthalmol. Vis. Sci. 37:1833-1839, 1996.

10. Crosson, C.E. Adenosine receptor activation modulates intraocular pressure in rabbits. J. Pharmacol. Exp. Ther. 273: 320-326, 1995. 
11. Crosson, C.E., and Gray, T. Modulation of intraocular pressure by adenosine agonists. J. Ocul. Pharmacol. 10: 379-383, 1994.

12. Crosson, C.E. Ocular hypotensive activity of the adenosine agonist (R)-phenylisopropyladenosine in rabbits. Curr. Eye. Res. 11:453-458, 1992.

13. Daines, B.S., Kent, A.R., McAleer, M.S., and Crosson, C.E. Intraocular adenosine levels in normal and ocularhypertensive patients. J. Ocul. Pharmacol. Ther. 19:113119, 2003.

14. Shearer, T.W., and Crosson, C.E. Adenosine A1 receptor modulation of MMP-2 secretion by trabecular meshwork cells. Invest. Ophthalmol. Vis. Sci. 43:3016-3020, 2002.

15. Zhong, Y., Yang, Z., Huang, W.C., and Luo, X. Adenosine, adenosine receptors and glaucoma: an updated overview. Biochim. Biophys. Acta. 1830:2882-2890, 2013.
16. Crosson, C.E., Sloan, C.F., and Yates, P.W. Modulation of conventional outflow facility by the adenosine A1 agonist N6-cyclohexyladenosine. Invest. Ophthalmol. Vis. Sci. 46: 3795-3799, 2005.

17. Lu, Z., and $\mathrm{Xu}, \mathrm{S}$. ERK1/2 MAP kinases in cell survival and apoptosis. IUBMB. Life. 58:621-631, 2006.

Received: October 28, 2015

Accepted: January 23, 2016

Address correspondence to: Dr. Rudolf A. Baumgartner Inotek Pharmaceuticals Corporation 91 Hartwell Avenue, Second Floor Lexington, MA 02421

E-mail: rbaumgartner@inotekpharma.com 